\title{
Mechanistic and Performance Studies on the Ligand-Promoted Ullmann Amination Reaction
}

\author{
Quintin A. Lo, ${ }^{\dagger}$ David Sale, ${ }^{\dagger}$ D. Christopher Braddock, ${ }^{\dagger}$ and Robert P. Davies ${ }^{*} \dagger \odot$
}

${ }^{\dagger}$ Department of Chemistry, Imperial College London, South Kensington, London SW7 2AZ, United Kingdom

${ }^{\ddagger}$ Process Studies Group, Jealott’s Hill Research Centre, Syngenta, Bracknell, Berkshire RG42 6EY, United Kingdom

\section{Supporting Information}

ABSTRACT: Over the last two decades many different auxiliary ligand systems have been utilized in the coppercatalyzed Ullmann amination reaction. However, there has been little consensus on the relative merits of the varied ligands and the exact role they might play in the catalytic process. Accordingly, in this work some of the most commonly employed auxiliary ligands have been evaluated for $\mathrm{C}-\mathrm{N}$ coupling using reaction progress kinetic analysis (RPKA)

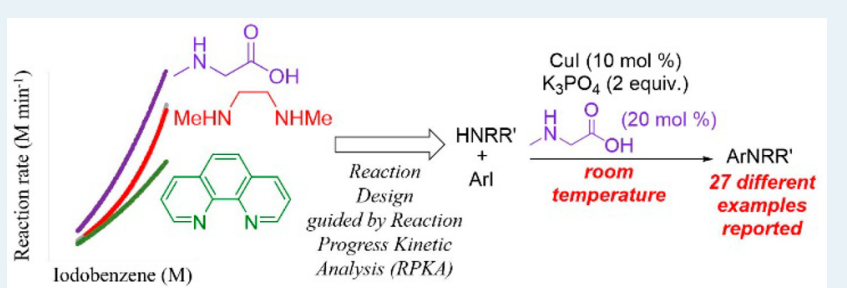
methodology. The results reveal not only the relative kinetic competencies of the different auxiliary ligands but also their markedly different influences on catalyst degradation rates. For the model Ullmann reaction between piperidine and iodobenzene using the soluble organic base bis(tetra- $n$-butylphosphonium) malonate (TBPM) at room temperature, $N$-methylglycine was shown to give the best performance in terms of high catalytic rate of reaction and comparatively low catalyst deactivation rates. Further experimental and rate data indicate a common catalytic cycle for all auxiliary ligands studied, although additional off-cycle processes are observed for some of the ligands (notably phenanthroline). The ability of the auxiliary ligand, base (malonate dianion), and substrate (amine) to all act competitively as ligands for the copper center is also demonstrated. On the basis of these results an improved protocol for room-temperature copper-catalyzed $\mathrm{C}-\mathrm{N}$ couplings is presented with 27 different examples reported.

KEYWORDS: copper, Ullmann reaction, $C-N$ bond coupling, amination, RPKA, organic bases, catalyst deactivation, room temperature

\section{INTRODUCTION}

The Ullmann $\mathrm{C}-\mathrm{N}$ cross-coupling reaction dates back to the early 1900s, where the use of stoichiometric amounts of copper and high reaction temperatures allowed for the coupling of aryl halides and amines. ${ }^{1}$ However, there were several drawbacks to this classical Ullmann reaction, where strong bases, long reaction times, and electron-deficient aryl substrates were required for the reaction to proceed. More recent developments include the use of bidentate ligands, such as 1,10phenanthroline (phen), which allowed the cross-coupling reaction to be carried out catalytically under milder reaction conditions (Scheme 1). ${ }^{2-12}$ Today, the modified Ullmann reaction is an important component in the toolbox of a synthetic organic chemist and has been employed in the synthesis of numerous bioactive compounds, natural products, and organic materials containing $\mathrm{C}-\mathrm{N}$ bonds. ${ }^{2-6,13-17}$

\section{Scheme 1. Modified Ullmann Amination Reaction}

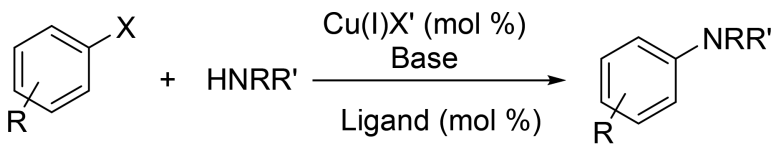

$X=$ halide
The low cost and toxicity of copper coupled with the use of cheap and readily available auxiliary ligands based on $\mathrm{N}$ and $\mathrm{O}$ atoms makes it an attractive alternative to the otherwise successful palladium-catalyzed Buchwald-Hartwig reaction. ${ }^{18-21}$ Historically, the mechanism of the Ullmann reaction has been the subject of much debate, with often contradictory interpretations of experimental and computational data in the literature. $^{22-25}$ This lack of understanding has hindered the development of more robust catalytic systems and the improvement of existing systems.

However, recent studies have all pointed toward the copper catalyst playing an important role in the activation of the aryl halide substrate, ${ }^{22,23}$ with spectroscopic evidence for an oxidative addition/reductive elimination pathway via a copper(III) intermediate. ${ }^{26-28}$ Despite these advances the role of the auxiliary ligand in the catalytic cycle remains relatively unexplored, with ligand optimization studies predominately based solely upon empirical final-yield figures, rather than any deeper kinetic or mechanistic insights.

Reaction progress kinetic analysis (RPKA) is a powerful methodology which can allow a simplified mechanism to be

\footnotetext{
Received: October 26, 2017

Revised: November 13, 2017

Published: November 30, 2017
} 
developed, taking into account catalyst inhibition/deactivation pathways and other reaction steps. ${ }^{29,30}$ However, commonly used inorganic bases in the Ullmann reaction such as $\mathrm{K}_{2} \mathrm{CO}_{3}$, $\mathrm{K}_{3} \mathrm{PO}_{4}$, and $\mathrm{Cs}_{2} \mathrm{CO}_{3}$ can obfuscate RPKA measurements due to their poor solubility in the polar aprotic solvents commonly used in this reaction. ${ }^{13,25}$ This prevents the collection of accurate kinetic data due to mass transfer effects of the base. Therefore, kinetic studies on copper-mediated coupling reactions have been limited in the past to reactions involving low-p $K_{\mathrm{a}}$ substrates such as the Goldberg reaction. ${ }^{31,32}$ However, we have recently shown that using soluble organic bases, such as bis(tetra- $n$-butylphosphonium) malonate (TBPM), can mitigate any base mass-transfer effects and thus enable the collection of much more accurate kinetic data in these systems. ${ }^{9,33}$ In addition, using TBPM gave excellent yields of cross-coupled products at temperatures as low as $0{ }^{\circ} \mathrm{C}$ with aryl iodides. $^{9,33}$ Studies on the TBPM-promoted ligand-free Ullmann reaction using the RPKA methodology carried out previously in our group have allowed us to recently propose a modified catalytic cycle (Scheme 2) and simplified steady-state rate law (eq 1 in Scheme 2) for this reaction and to explore in more depth possible catalyst deactivation pathways. First-order

Scheme 2. Proposed Reaction Mechanism for the $\mathrm{Cu}$ Catalyzed Cross Coupling Reaction between 1 and 2 under Ligand-Free Conditions ${ }^{33}$

(1): Rate $=K^{\prime}[1][2][\mathrm{Cu}]_{\text {total }} \quad K^{\prime}=K_{1}^{-1} K_{2} K_{3} K_{4}$
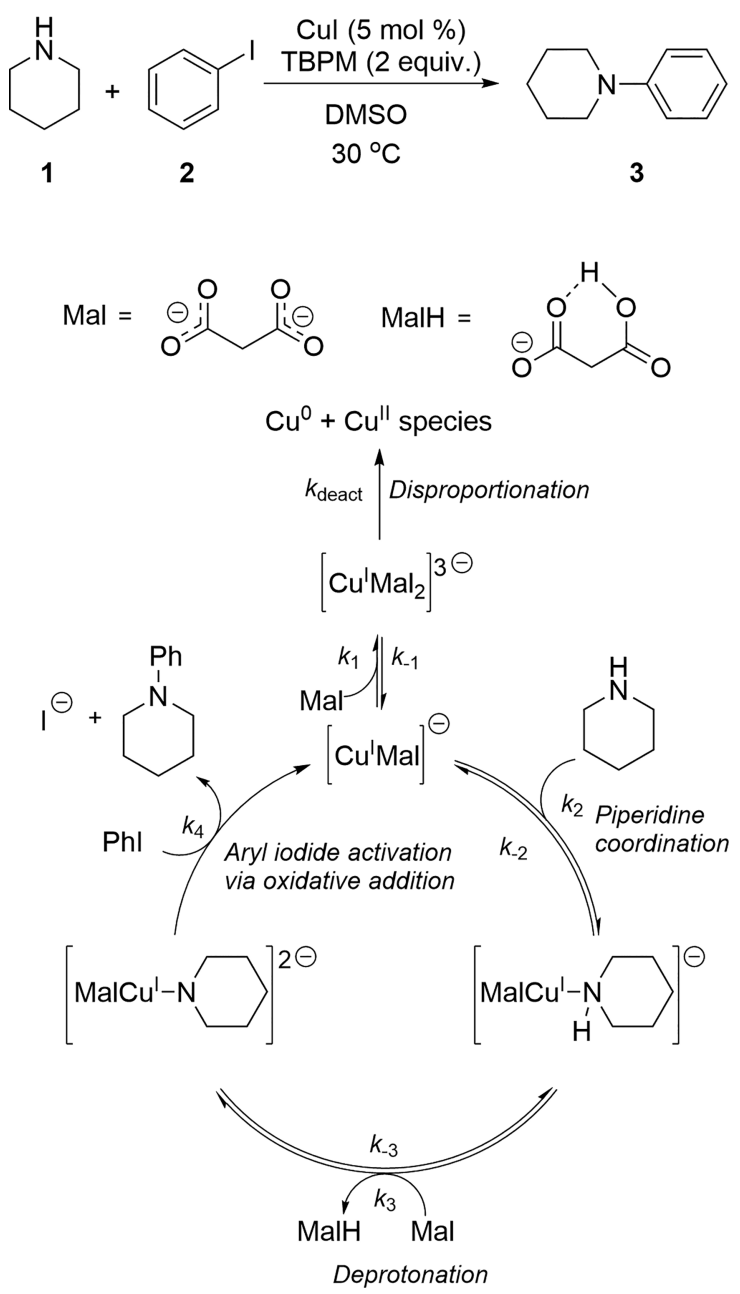

kinetics were observed with respect to $[\mathbf{1}],[2]$, and $[\mathrm{Cu}]_{\text {total }}$, coupled with negative dependence on [TBPM.$^{33}$ This negative dependence on base was attributed to the formation of off-cycle copper(I) species. The key role of the base in Ullmann and Goldberg reactions and its ability to competitively bind to the copper(I/III) metal center, has also been highlighted in recent studies by Sharma ${ }^{27}$ and Nguyen. ${ }^{34}$

In this work, we extend our studies on the mechanism of the catalytic Ullmann reaction by kinetically profiling a wide range of commonly used auxiliary ligands in the cross-coupling of piperidine and iodobenzene using TBPM as the base. These results allow us to move beyond just looking at product yields and compare for the first time the influence of the auxiliary ligand on the rate of reaction, rate dependence in substrates, and catalyst deactivation/inhibition pathways.

\section{RESULTS AND DISCUSSION}

2.1. Ligand Screening. In continuation of our earlier work, the reaction between piperidine (1) and iodobenzene (2) was chosen as the model system for kinetic study. Vigorous air- and moisture-free conditions were employed. Piperidine is of particular interest, as it has been cited as one of the most frequently used nitrogen heterocycles in U.S. FDA approved drugs. ${ }^{35}$ Under auxiliary-ligand-free conditions, we observed TBPM to give good reactivity ( $98 \%$ yield) at room temperature relative to inorganic bases such as $\mathrm{K}_{2} \mathrm{CO}_{3}, \mathrm{~K}_{3} \mathrm{PO}_{4}$, and $\mathrm{Cs}_{2} \mathrm{CO}_{3}$ (all $<6 \%$ yield). This can be attributed to the solubility of and dissociation of TBPM in DMSO at room temperature ${ }^{9}$ in comparison to inorganic bases and the ability of the malonate component of TBPM to serve as a ligand as well as a base. ${ }^{33}$ Initial screenings were carried out using a diverse range of auxiliary ligands, many of which are taken from successful Ullmann coupling protocols in the literature (Scheme 3 ). Although $\mathrm{Ma}$ and co-workers have recently reported a series of oxalic diamide ligands that can activate aryl bromides and chloride substrates in the Ullmann reaction, ${ }^{36-41}$ these had to be omitted from this study due to their very poor solubility in DMSO at room temperature.

The rate of reaction was monitored for each auxiliary ligand using reaction calorimetry. To verify that the calorimetric data do correctly correlate with the progress of the reaction, a sample reaction (using ethylene glycol, L14) was monitored using ${ }^{1} \mathrm{H}$ NMR spectroscopy and the product yield directly compared against that determined using heat flow experiments. Close agreement between heat flow and NMR conversions was observed (see Figure 1).

The determined enthalpy of reaction $\Delta H_{\mathrm{rxn}}$ for all experiments in this study was consistent within $\pm 5 \%\left(\Delta H_{\text {rxn }}=170.5\right.$ $\left.\pm 8.5 \mathrm{~kJ} \mathrm{~mol}^{-1}\right)$. Varying the initial substrate concentrations did not influence the calculated enthalpy of reaction $\Delta H_{\mathrm{rxn}}$. These experiments therefore validate the use of reaction calorimetry as a tool to study the ligand-promoted Ullmann reaction.

Complete (100\%) conversion of iodobenzene was obtained for all reactions. The yield of the desired cross-coupled product 3 in each case was in excess of $98 \%$ with approximately $2 \%$ yield of benzene side product also observed. In no cases were any aryl iodide to ligand couplings observed (even with ligands containing $\mathrm{NH}$ functionalities). The benzene is most likely formed from hydrodehalogenation of the iodobenzene, with similar hydrodehalogenation side products already reported in the literature for other copper(I)-catalyzed protocols. ${ }^{33,42-44}$ Variation in the type of auxiliary ligand had no effect on the amount of benzene formed, and the auxiliary ligand can thus be 
Scheme 3. Kinetic Studies on the Ullmann Amination Reaction between 1 and 2 Using Various Auxiliary Ligands ${ }^{a}$

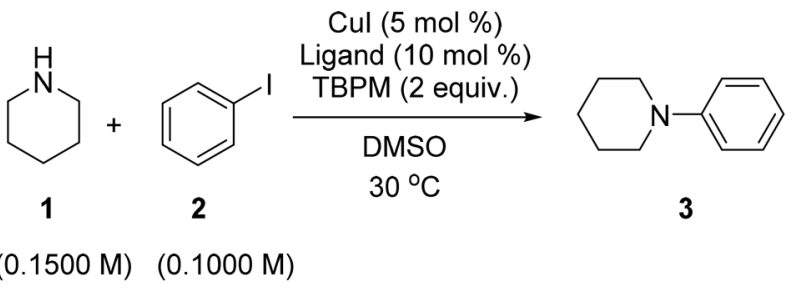

\section{$N, N$ ligands}
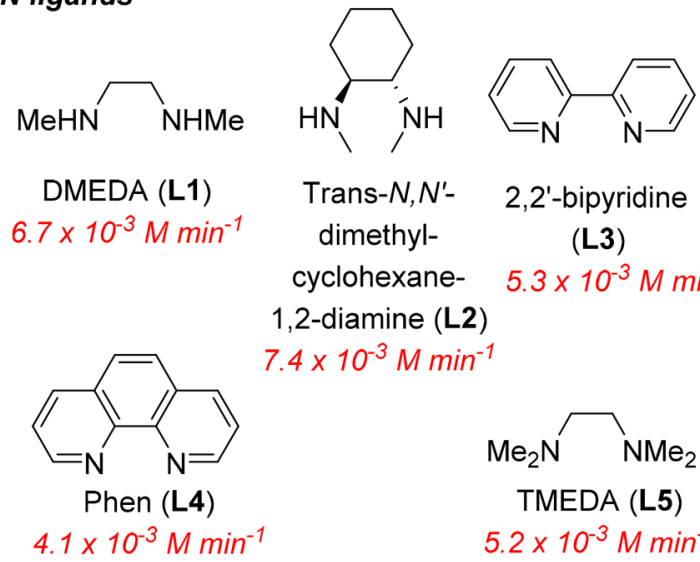

Trans- $N, N^{\prime}-$ dimethyl-

2,2'-bipyridine

(L3)

cyclohexane- $5.3 \times 10^{-3} \mathrm{M} \mathrm{min}^{-1}$

1,2-diamine (L2)

$7.4 \times 10^{-3} \mathrm{M} \mathrm{min}^{-1}$

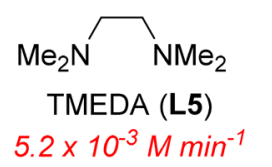

\section{$N, O$ ligands}<smiles>CNCC(=O)O</smiles><smiles>CN(C)CC(=O)O</smiles><smiles>O=C(O)c1ccccn1</smiles>

$N$-methyl$N, N^{\prime}$-dimethylglycine (L7)

2-picolinic acid

(L8) $7.4 \times 10^{-3} \mathrm{M} \mathrm{min}^{-1} 6.0 \times 10^{-3} \mathrm{M} \mathrm{min}^{-1} 5.6 \times 10^{-3} \mathrm{M} \mathrm{min}^{-1}$

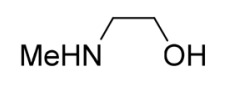

2-methylaminoethanol (L11) $5.5 \times 10^{-3} \mathrm{M} \mathrm{min}^{-1}$
L-proline (L9)

D-proline (L10)

$6.4 \times 10^{-3} \mathrm{M} \mathrm{min}^{-1}$

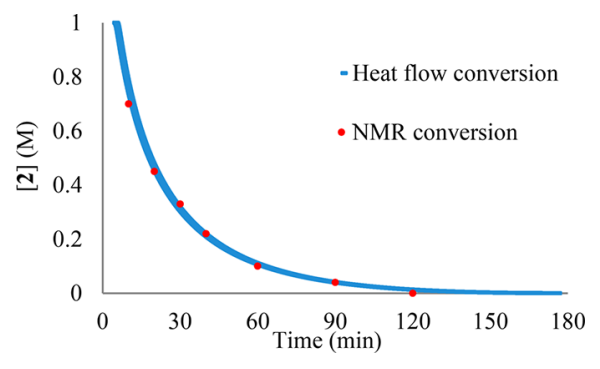

Figure 1. Comparison between heat flow and ${ }^{1} \mathrm{H}$ NMR conversions.

negligible relative to the $\mathrm{C}-\mathrm{N}$ cross-coupling reaction and can thus be ignored.

Heat flow vs time plots were collected using reaction calorimetry in the presence of each auxiliary ligand as well as under auxiliary-ligand-free conditions (Figure S2 in the Supporting Information). These highlight the difference in reactivity as seen by the variations in maximum heat flow. The heat flow plots were processed to give the initial rate of reaction for each studied auxiliary ligand (Scheme 3). Triphenylphosphine (L16) and the tetradentate Salen ligand (L17) were also studied but furnished product yields of only 13 and $48 \%$, respectively; this led to large potential errors in their calorimetrically determined reaction rates, and hence they have not been included in Scheme 3 .

Most of the auxiliary ligands studied gave a faster initial rate of reaction relative to the auxiliary-ligand-free reaction. However, L4 and L13 exhibited slower initial reaction rates and L3, L5, L12, and L15 had no observable effect on the reaction rate. Changing the ligand loadings of this latter set of ligands also had no effect on the initial reaction rates (Figure S3 in the Supporting Information). These results reflect the competitive nature of the binding of the auxiliary ligand and the malonate dianion for copper(I), with the equilibrium sitting far on the side of the malonate-coordinated species for L3, L5, L12, and L15.

Overall the ligand screening results show that auxiliary ligands containing secondary amine functionalities give the best reactivity. Tertiary amines are less active in promoting the reaction, as can be seen from the comparisons of L1 with L5 and of L6 with L7. A key factor in these observations is likely to be the steric bulk of the ligand. ${ }^{23}$ In addition, auxiliary ligands with pyridine or alcohol/phenol functionalities display little or no ligand-based acceleration effect. While some similar patterns in ligand preferences have been reported in the literature, ${ }^{8-10,45-48}$ these have been based on final yield measurements and as far as we are aware have not incorporated any kinetic measurements.

2.2. Catalyst Deactivation. The collection of experimental rate data continuously over the course of a reaction combined with RPKA methods allows additional mechanistic insights over and above the initial rate calculations to be made. ${ }^{29,30}$ All reactions reported herein were monitored in situ using reaction calorimetry to produce "graphical rate equations". 29,30 Only data between 20 and $80 \%$ conversions are displayed to limit any inaccuracies as the limiting substrate (2) approaches zero and to eliminate any heat transfer effects. Representative examples of these plots are shown in Figure 2 (see the Supporting Information for all plots over all conversion values). It is immediately apparent that, in addition to differing initial rates, the curvature of these plots varies. Plots of auxiliary ligands with two $\mathrm{NH}$ groups ( $\mathbf{L} 1$ and $\mathbf{L 2}$ ) have a higher curvature than the 


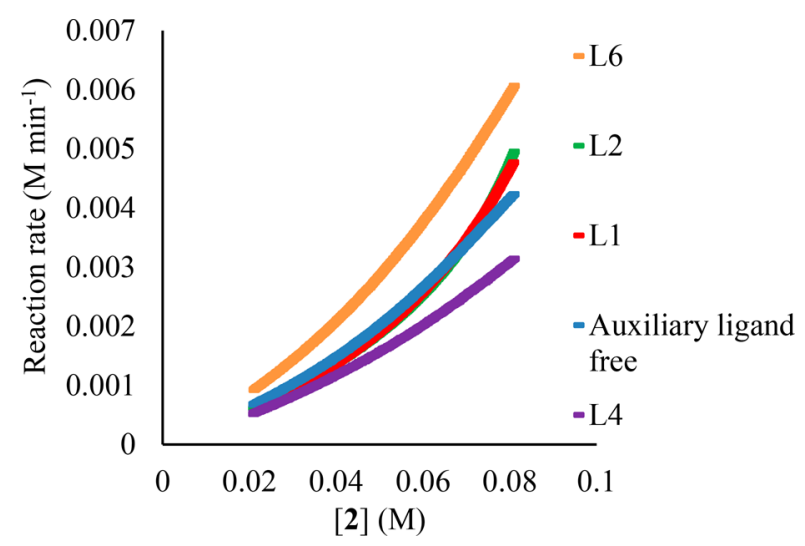

Figure 2. Graphical rate equations for experiments using L1, L2, L4, L6, and auxiliary-ligand-free reactions using the conditions shown in Scheme 3.

other plots. Thus, although L2 exhibits an initial rate identical with that of L6 (Scheme 3), this rate drops off far more quickly as the reaction progresses. This is indicative of differences in the mechanisms or the presence of alternative reaction pathways such as catalyst deactivation or inhibition. In order to probe these differences, information regarding catalyst deactivation and the rate dependence in substrates was sought through different and same excess experiments. For all experiments reported herein, $\mathbf{1}$ is in excess while $\mathbf{2}$ is the limiting substrate.

Same excess experiments were carried out in the presence of ligands L1, L4, and L6 to determine if catalyst deactivation occurs over the course of the reaction (Figure 3). These ligands were chosen as they differed the most from the auxiliary-ligandfree reaction, exhibiting either faster or slower reaction rates or, in the case of $\mathbf{L} \mathbf{1}$, a different reaction profile. The same excess experiment involved a reduction in $[\mathbf{1}]_{0},[\mathbf{2}]_{0}$, and $[\mathrm{TBPM}]_{0}$, while keeping their difference in concentration the same (i.e., $[e]=0.0500 \mathrm{M})$. This is equivalent to starting the same reaction but at different starting points. Therefore, the lack of overlap between the same excess experiment (orange, Figure 3) and the standard experiment (blue, Figure 3) confirms that catalyst deactivation or product inhibition does indeed occur. Product inhibition was ruled out by carrying out an extra same excess experiment involving the addition of product 3 and byproducts 4 and $\mathbf{5}$ into the reaction mixture (green, Figure 3). Overlap between the second (green) and first (blue) same excess experiment curves shows that any catalyst inhibition caused by product coordination, solution conductivity changes caused by the formation of tetra- $n$-butylphosphonium iodide, or protonation of piperidine by monoanionic malonic acid are all negligible in this reaction regardless of the ligand used. Thus, catalyst decomposition is the most likely deactivation process.

The gap between the same excess experiment and the standard experiment in Figure 3 is noticeably larger for L1 than for L4, L6, and auxiliary-ligand-free reactions. At the arbitrary value of $[2]=0.4 \mathrm{M}$, the reaction rate was $60 \%$ lower with L1 in comparison to $30 \%$ lower with L6 and $12 \%$ lower with L4. Significantly increased catalyst deactivation is therefore occurring in the presence of $\mathbf{L} \mathbf{1}$, while a reduction in catalyst deactivation occurs with L4. On the basis of these studies, L6 would seem to provide the best balance with high rate of reaction and comparatively low catalyst deactivation.

A multiple substrate addition protocol was carried out to further verify the presence of catalyst deactivation: $0.1000 \mathrm{M}$ of
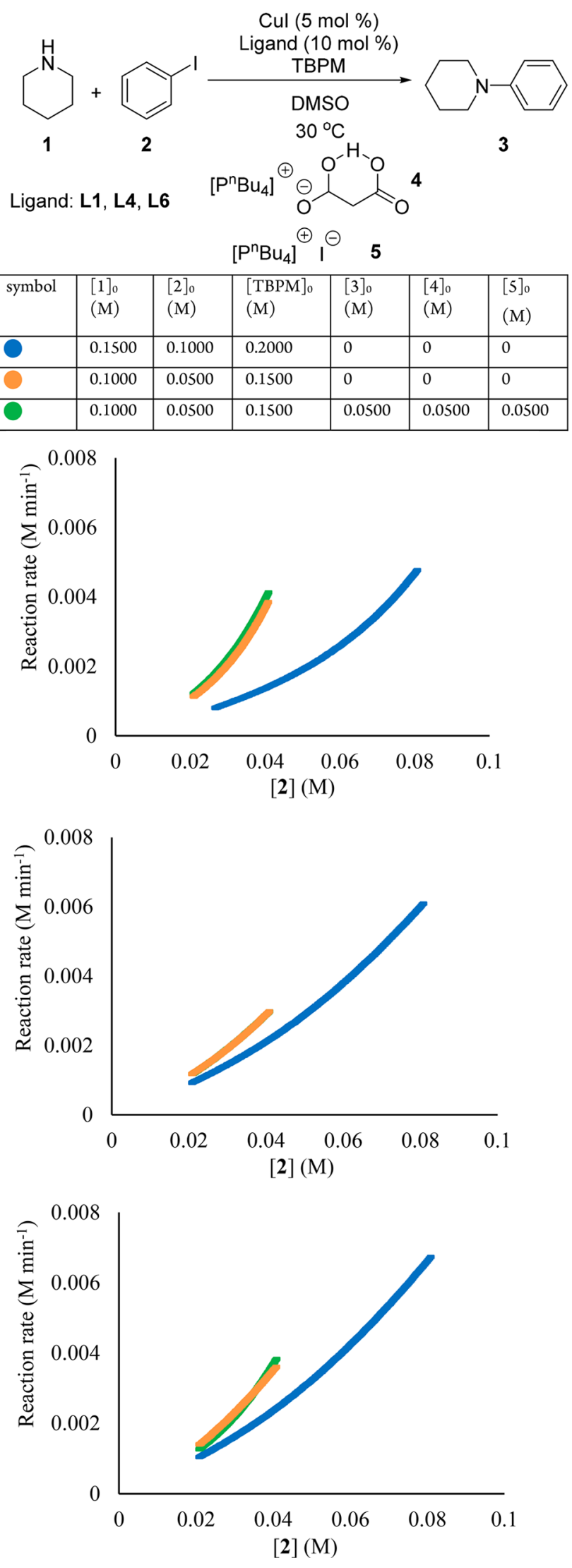

Figure 3. Graphical rate equations for experiments carried out using the same excess protocol with L1 (top), L4 (middle), and L6 (bottom). See the Supporting Information for full plots. 
$[1]_{0}$ and $0.0500 \mathrm{M}$ of $[2]_{0}$ in the presence of $10 \mathrm{~mol} \%$ of ligand (L1, L2, L4, or L6) was initiated with $5 \mathrm{~mol} \%$ of CuI. Once this first reaction had reached completion (i.e., negligible heat flow was detected by the calorimeter), a further solution of 1 and $2(0.500 \mathrm{mmol}$ each in $200 \mu \mathrm{L}$ of DMSO) was added to the reaction mixture to restart the reaction. A third reaction was subsequently started once the second reaction had ended. If no catalyst deactivation occurs, each subsequent reaction should be marginally faster than the previous one owing to lower [TBPM]. This is due to the negative dependence in [TBPM] caused by the formation of off-cycle copper(I) dimalonate species (Scheme 2) as determined previously. ${ }^{33}$ However, this was not the case, as each subsequent reaction was significantly slower than the previous one. An example of this with $\mathbf{L 1}$ is shown in Figure 4 (for other ligands see Figure S5 in the

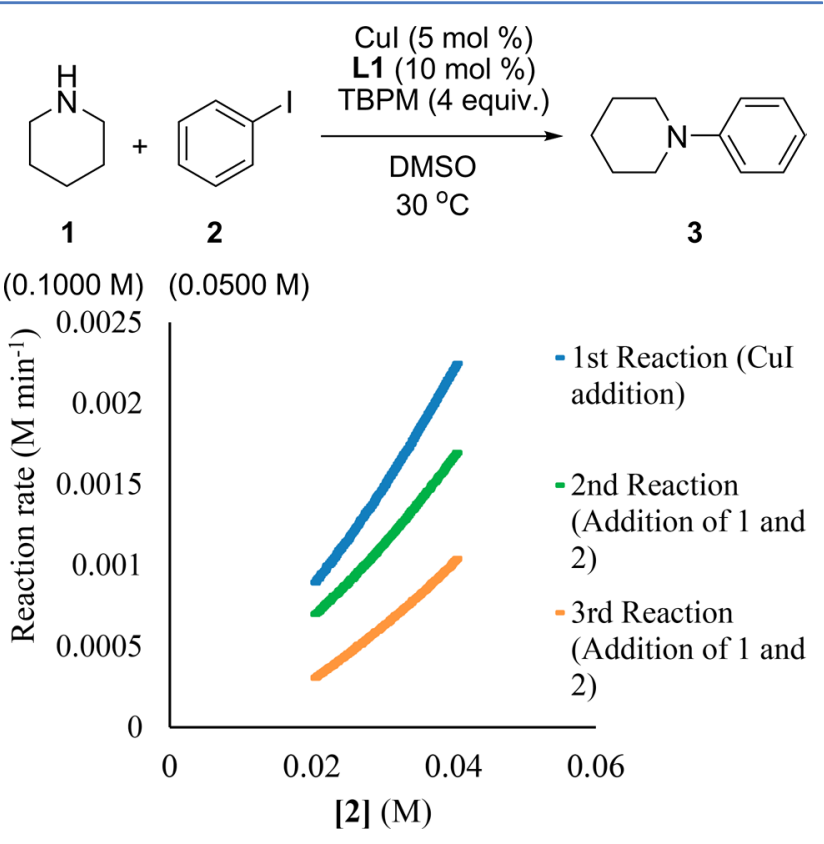

Figure 4. Graphical rate equations for each sequential reaction with L1.

Supporting Information). Therefore, catalyst deactivation is indeed occurring. The change in catalyst concentration due to an increase in reaction volume is negligible, as it is much smaller than the change in rate of reaction. Due to catalyst deactivation, the third and final reaction gave only $75 \%$ yield of 3 for all four auxiliary ligands studied.

The stability of the prospective copper(I) intermediates in the presence of these ligands was also investigated by addition of the respective ligand to freshly prepared copper(I) piperidide $^{49}$ in $\left[\mathrm{D}_{6}\right] \mathrm{DMSO}$ at room temperature under an inert atmosphere. Thus, addition of $\mathbf{L} \mathbf{1}$ to copper(I) piperidide resulted in total decomposition of the copper piperidide complex within $2 \mathrm{~h}$ (determined using ${ }^{1} \mathrm{H}$ NMR with mesitylene as an internal standard). However, when L4 was added (or under auxiliary-ligand-free conditions), less than $1 \%$ complex decomposition was observed over a similar time period. Finally, addition of L6 gave approximately 60\% copper(I) piperidide decomposition after $2 \mathrm{~h}$. For each of these above reactions, an equivalence of iodobenzene was subsequently added to the resultant solution, and the mixture was stirred for $24 \mathrm{~h}$ at $80{ }^{\circ} \mathrm{C}$. Conversion to the $\mathrm{C}-\mathrm{N}$ coupled product 3 was determined using ${ }^{1} \mathrm{H}$ NMR and found to be $0 \%$ for $\mathbf{L} 1$ and $\mathbf{L 2}$, $15 \%$ for $\mathbf{L 4}$, and $43 \%$ for $\mathbf{L 6}$. These results are consistent with the calorimetric data, showing L6 to confer the best balance between high rate of reaction and low rate of catalyst deactivation. The lack of any conversion at all for the L1- and L2-treated samples suggests that catalyst deactivation under these conditions is irreversible. However, a reversible catalyst deactivation process cannot be ruled out under the catalytic conditions and further studies are still required to clarify the exact mechanism of catalyst deactivation.

2.3. Rate Dependence in Substrates. Rate dependence in substrates was determined using RPKA methods for both L4 and L6. Reactions with these two auxiliary ligands both showed reasonable catalyst stability (vide supra) and allowed comparison between a ligand that gives a faster (L6) and slower (L4) rate of reaction relative to that of the auxiliaryligand-free reaction. A series of different excess experiments were carried out to determine the rate dependence of $[1]_{0}$, $[\mathbf{2}]_{0},[\mathrm{TBPM}]_{0}$, and $[\mathrm{Cu}]_{\text {total }}$.

Decreasing $[2]_{0}$ from 0.1000 to $0.0500 \mathrm{M}$ while keeping the concentrations of other substrates and catalyst the same led to a corresponding decrease in the reaction rate (Figure 5; for full plots refer to Figure S6 in the Supporting Information) in the presence of either L4 and L6. Moderately good overlap between the normalized rate equations when the reaction rate was normalized by [2] is indicative of first-order dependence with respect to [2] (Figure 5). This is consistent with what was observed with the auxiliary-ligand-free reaction. ${ }^{33}$

The rate dependence in $[\mathrm{Cu}]_{\text {total }}$ was probed next. Lowering $[\mathrm{Cu}]_{\text {total }}$ from 10 to $5 \mathrm{~mol} \%$ gave a corresponding decrease in the reaction rate regardless of the auxiliary ligand used (Figure 6; for full plots refer to Figure S7 in the Supporting Information). Normalized rate equations revealed a first-order dependence in $[\mathrm{Cu}]_{\text {total }}$ with either L4 or L6 (Figure 6).

Negative dependence in [TBPM] was observed in the presence of both L4 and L6, with increasing $[\mathrm{TBPM}]_{0}$ leading to slower reaction rates (Figure $S 8$ in the Supporting Information). Negative dependence in [TBPM] can be attributed to the formation of off-cycle copper(I) dimalonate species which in turn can undergo disproportionation to copper(0) and copper(II) at room temperature even under inert atmospheres (see Scheme 2). ${ }^{33,50,51}$

Positive noninteger dependence on [1] was observed using the difference excess protocol with L4 or L6 (Figure S11 in the Supporting Information). In comparison, a first-order dependence on [1] was observed under ligand-free conditions. ${ }^{33}$ This can be rationalized by the presence of off-cycle equilibria involving the auxiliary ligand (vide infra). However, reactions involving a large excess of $[\mathbf{1}]_{0}$ move toward first order in $[\mathbf{1}]$ with observed rate values that are independent of the auxiliary ligand employed (see Figures S12 and S13 in the Supporting Information). We believe this is due to displacement of the auxiliary ligand at very high $[\mathbf{1}]$ to give an on-cycle $\left[\mathrm{Cu}\left(\mathrm{C}_{5} \mathrm{H}_{10} \mathrm{~N}\right)\left(\mathrm{C}_{5} \mathrm{H}_{10} \mathrm{NH}\right)\right]$ species. This behavior is indicative of competitive ligand binding at the copper center.

Taken together, these different excess experiments with L4 or L6 showed that, at $\leq 0.3 \mathrm{M}$ of $[\mathbf{1}]_{0},[\mathbf{2}]_{0},[\mathrm{TBPM}]_{0}$, and $[\mathrm{Cu}]_{\text {total, }}$ all reaction components influence the reaction rate. First-order dependence on $[\mathbf{2}]$ and $[\mathrm{Cu}]_{\text {total, a positive }}$ dependence on $[\mathbf{1}]$, and a negative dependence on [TBPM] were observed.

2.4. Rate Dependence in Ligand. The effect of auxiliary ligand loading on the initial rate of reaction for several of the ligands is illustrated in Figure 7. The bar chart illustrates how 
<smiles>C1CCNCC1</smiles><smiles>Ic1ccccc1</smiles>

2

$$
\text { Cul }(5 \mathrm{~mol} \%)
$$

Ligand (10 mol \%)

TBPM ( 2 equiv.)

DMSO

$30^{\circ} \mathrm{C}$

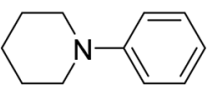

3

Ligand: L4 or L6

\begin{tabular}{|l|l|l|l|}
\hline Symbol & {$[\mathbf{1}]_{0}(\mathrm{M})$} & {$[\mathbf{2}]_{0}(\mathrm{M})$} & {$[e]_{0}$} \\
\hline & 0.1500 & 0.1000 & 0.0500 \\
\hline & 0.1500 & 0.0500 & 0.1000 \\
\hline
\end{tabular}
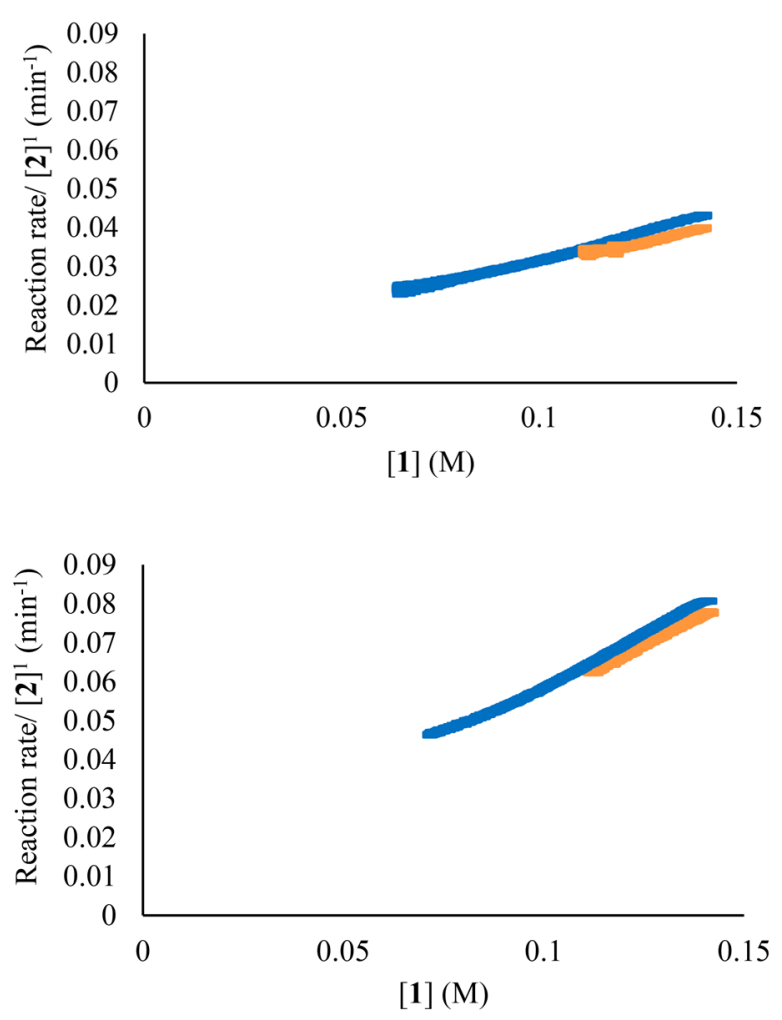

Figure 5. Normalized graphical rate equations indicating first order in [2] for L4 (top) and L6 (bottom). [e] = "excess" $=[\mathbf{1}]_{0}-[\mathbf{2}]_{0}$. See the Supporting Information for full graphical rate plots.

positive dependence on $\mathbf{L 1}, \mathbf{L 2}$, and $\mathbf{L 6}$ was observed in the range of $5-20 \mathrm{~mol} \%{ }^{52}$

Variation in L3 loadings did not influence the reaction rate, which remained essentially identical with that of the auxiliaryligand-free reaction (Figure 7). This can be explained by the presence of an excess of malonate dianion from the base which acts competitively as a ligand for the copper center with L3, with the equilibrium siting predominately on the side of the malonate coordinated species.

Increasing L4 loadings led to a slower rate of reaction, which can be attributed to its higher binding affinity toward copper(I) (in comparison to L3), ${ }^{53}$ combined with a tendency to form the unreactive separated ion-pair complexes $\left[\mathrm{Cu}(\mathbf{L} 4)_{2}\right]^{+}[\mathrm{Cu}-$ (piperidide) $\left.{ }_{2}\right]^{-}$as shown in section 2.5. The formation of such an off-cycle species is already well-known for similar L4-ligated $\mathrm{Cu}(\mathrm{I})$ species $^{2,32,34,43,49,54}$ and explains the observed negative dependence on $\mathbf{L} 4$ and slower rate of reaction in comparison to the auxiliary-ligand-free system.

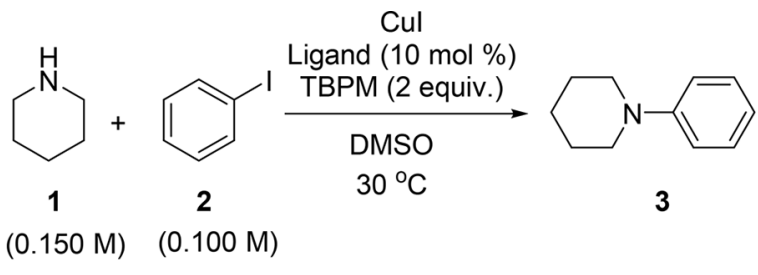

Ligand: L4 or L6

\begin{tabular}{|l|l|}
\hline Symbol & {$[\mathrm{Cu}]_{\text {total }}(\mathrm{M})$} \\
\hline & $2.50 \times 10^{-3}$ \\
\hline & $5.00 \times 10^{-3}$ \\
\hline
\end{tabular}
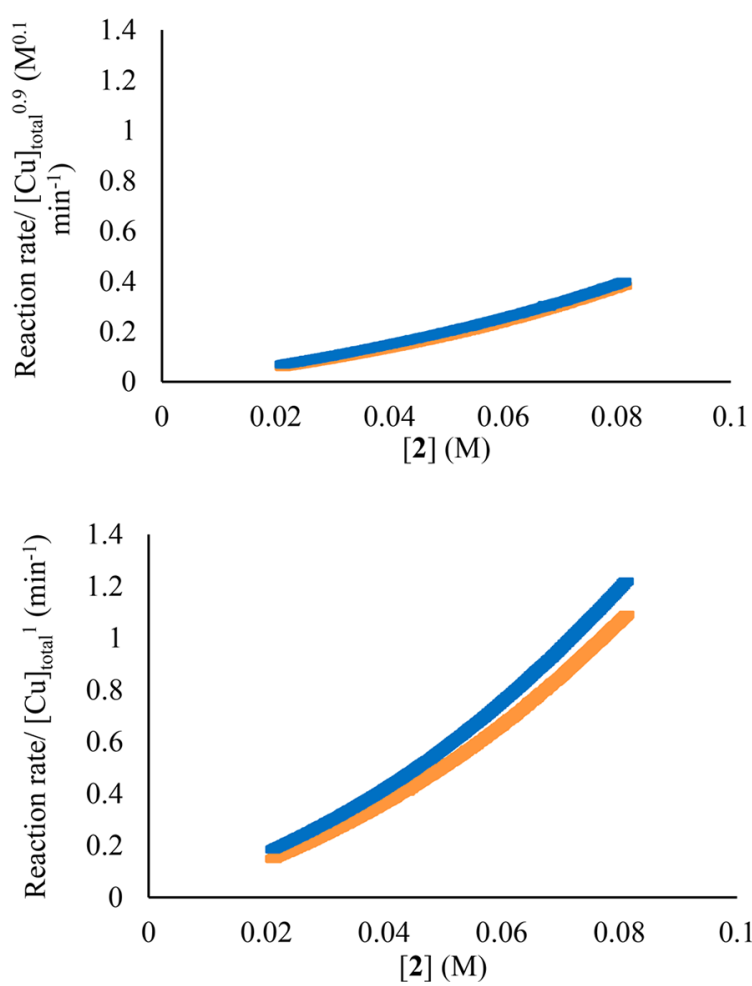

Figure 6. Normalized graphical rate equations indicating first order in $[\mathrm{Cu}]_{\text {total }}$ for $\mathbf{L 4}$ (top) and L6 (bottom). Full graphical rate plots are available in the Supporting Information.

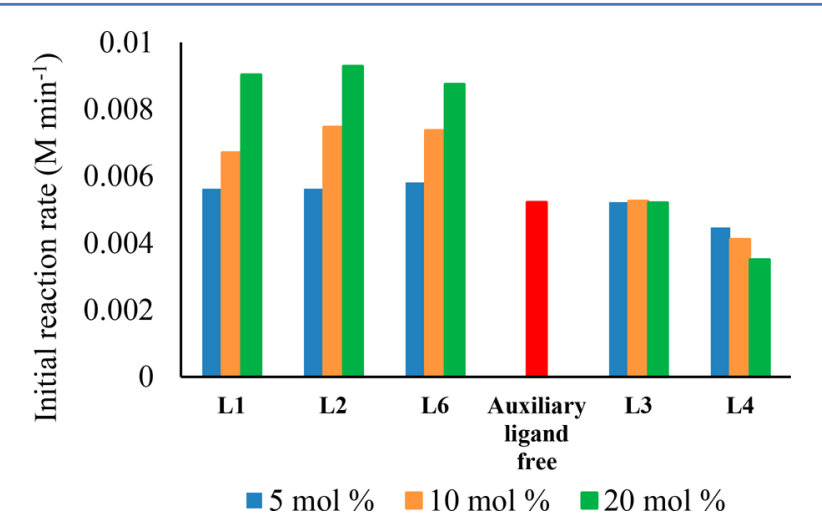

Figure 7. Bar chart illustrating the reaction rate at different auxiliary ligand loadings.

2.5. Catalyst Speciation with Auxiliary Ligand. Attempts were made to study the catalyst speciation in the 
presence of auxiliary ligands L1, L2, L4, and L6. However, on addition of L1, L2, or L6 to copper(I) complexes (piperidide, iodide, or tert-butoxide) in $\left[\mathrm{D}_{6}\right] \mathrm{DMSO}$, in all cases decomposition of the resultant species was observed (to copper(0) and copper(II) salts) even under vigorous air- and moisture-free conditions. However, similar studies with L4 proved more successful, giving stable complexes amenable to characterization by ${ }^{1} \mathrm{H}$ NMR spectroscopy.

Thus, a series of NMR experiments were carried out with L4 to probe the formation and stability of the potential ionseparated species $\left.\left[\mathrm{Cu}(\mathbf{L} 4)_{2}\right]^{+}[\mathrm{Cu} \text { (piperidide })_{2}\right]^{-}$. Lithium bis(piperidido)cuprate(I) was prepared from copper(I) iodide and lithium piperidide, ${ }^{49}$ and its ${ }^{1} \mathrm{H}$ NMR spectrum directly compared to that of a mixture of copper(I) piperidide and L4 (1:1 ratio). In both cases, identical shifts were observed for the piperidide resonances (Figure S16 in the Supporting Information). Moreover, addition of $\mathbf{L} 4$ and piperidine to $\left[\mathrm{CuO}^{\mathrm{t}} \mathrm{Bu}\right]_{4}$ in THF gave a waxy red solid (see the Supporting Information), which was analyzed by ${ }^{1} \mathrm{H}$ NMR spectroscopy in $\left[\mathrm{D}_{6}\right] \mathrm{DMSO}$ (Figure S16) to give once more an identical spectrum, again supporting the formation of $\left[\mathrm{Cu}(\mathrm{L} 4)_{2}\right]^{+}[\mathrm{Cu}-$ (piperidide) $\left.{ }_{2}\right]^{-}$during the cross-coupling reaction. These findings are consistent with previous literature reports for this ligand. $^{31,32,43,54}$

Addition of iodobenzene (2) to either lithium bis(piperidido)cuprate(I) or $\left.\left[\mathrm{Cu}(\mathbf{L} 4)_{2}\right]^{+}[\mathrm{Cu} \text { (piperidide) })_{2}\right]^{-}$gave no further reaction, and no $\mathrm{N}$-phenylpiperidine (3) was observed. This supports the notion that these ion-pair species are unreactive in the Ullmann coupling reaction. In addition, all these cuprate species were observed to be stable at room temperature under inert atmospheres for approximately 2 days.

2.6. Aryl Halide Activation. The aryl halide activation step was investigated using 2-(allyloxy)iodobenzene as a radical probe to determine if radical intermediates are present under the reaction conditions used in this study. The presence of radical intermediates will cause the radical probe to undergo rapid ring closure via a 5-exo-trig process $\left(k=9.6 \times 10^{9} \mathrm{~s}^{-1}\right.$ in DMSO), and the resulting methyl radical generated will subsequently react with the nucleophile. ${ }^{55}$ However, in the absence of such radical intermediates $\mathrm{C}-\mathrm{N}$ bond formation would be observed. The reaction between piperidine and the radical probe with $\mathbf{L 1}, \mathbf{L 2}, \mathbf{L 4}$, or $\mathbf{L 6}$ gave only the $\mathrm{C}-\mathrm{N}$ coupled product (Scheme 4), suggesting that aryl halide activation proceeds via oxidative addition/reductive elimination

Scheme 4. Reaction between 2-(Allyloxy)iodobenzene and Piperidine in the Presence of L1, L2, L4, or L6 To Determine if the Aryl Halide Activation Step Proceeds via Radical Intermediates
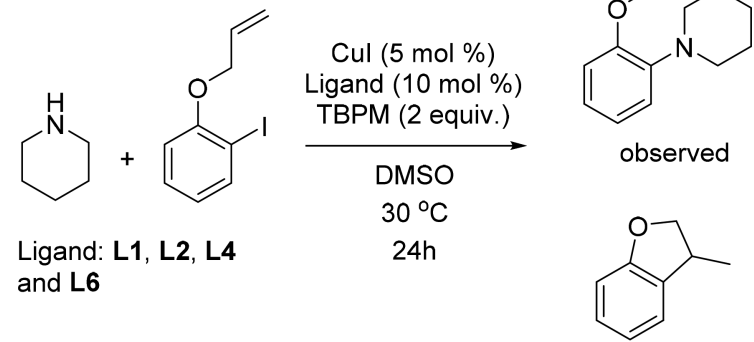

not observed through a copper(I)/copper(III) mechanism. This is consistent with previous work carried out by Hartwig et al. ${ }^{54}$

2.7. Reaction Design toward Improved Performance. One of the aims of this work is to allow a more systematic and informed quality-by-design approach to catalyst and auxiliary ligand selection in copper-catalyzed protocols. Hence, we sought to apply our findings to room-temperature $\mathrm{C}-\mathrm{N}$ couplings using common inorganic bases which are currently very challenging with existing systems.

Initially several inorganic bases were tested for the crosscoupling of a model system (benzylamine and iodobenzene), and $\mathrm{K}_{3} \mathrm{PO}_{4}$ was shown to give excellent yields using a higher catalyst loading of $10 \%$ (Table S1 in the Supporting Information). Although TBPM is a significantly superior base under these conditions, it was thought that its lack of commercial availability would make it less attractive to the academic and industrial communities at present. Unfortunately, we have been unable to carry out a full kinetic evaluation of the reaction using $\mathrm{K}_{3} \mathrm{PO}_{4}$ due to mass transfer effects of the base. However, auxiliary ligand screening showed a good correlation between the achieved product yield with $\mathrm{K}_{3} \mathrm{PO}_{4}$ (Table S2 in the Supporting Information) and the observed reaction rates with TBPM (section 2.1). In addition, mechanisms equivalent to Scheme 2 have been proposed for Ullmann couplings employing $\mathrm{K}_{3} \mathrm{PO}_{4}$, and recent studies have shown that phosphonate ions can act as both a ligand and a base in the catalytic cycle (thus being analogous to the observed behavior of the malonate anion in TBPM). ${ }^{26,27}$

On the basis of the RPKA results $N$-methylglycine (L6) was chosen as the auxiliary ligand, showing the best balance between fast initial rate of reaction and low levels of catalyst deactivation. A slight excess of amine (1.5 equiv) was employed to drive the reaction forward to the catalyst resting state $\left(\mathbf{L C u N R}_{2}\right)$ and prevent catalyst deactivation (Scheme 2). ${ }^{33}$

The reaction scope was extended to a variety of amines and aryl iodides: 27 examples in total (Table 1). The product yields obtained all compare favorably with those of room-temperature copper(I) protocols (where known ${ }^{11,56}$ ). Most substrate combinations gave high yields $(75 \%+)$, although electrondeficient $(\mathbf{1 1} \mathbf{g}-\mathbf{i})$ and sterically bulky $(\mathbf{1 1} \mathbf{j}, \mathbf{k})$ amines gave poorer conversions. In addition, ortho-substituted aryl iodides $(11 \mathbf{u}-\mathbf{x})$ gave no or little conversion, showing that the system is highly sensitive to steric bulk at this position. In no case was any coupling of the $\mathrm{N}$-methylglycine with the aryl iodide observed.

Given the mildness of the reaction conditions and its efficiency, the reaction was also carried out on a larger scale to test its scalability. No significant effects on the product yields were observed on scale-up ( $10 \mathrm{mmol}$ scale): $85 \%$ yield for $11 \mathrm{a}$ and $70 \%$ for $\mathbf{1 1 b}$.

\section{CONCLUSIONS}

An evaluation of some of the most commonly employed auxiliary ligands for application in the copper(I)-catalyzed Ullmann amination reaction has been undertaken. The model reaction between piperidine and iodobenzene in the presence of the soluble organic base bis(tetra- $n$-butylphosphonium) malonate (TBPM) has been adopted due to its high performance at room temperature and the absence of any mass transfer effects of the base. Kinetic studies using reaction calorimetry and RPKA methodology have revealed the relative catalytic reaction rates for each of these ligand systems as well as their different influences on catalyst deactivation. The rates 
Table 1. Coupling Reaction between Amines and Aryl Iodides with $\mathrm{K}_{3} \mathrm{PO}_{4}$ and $\mathrm{L}^{a}$

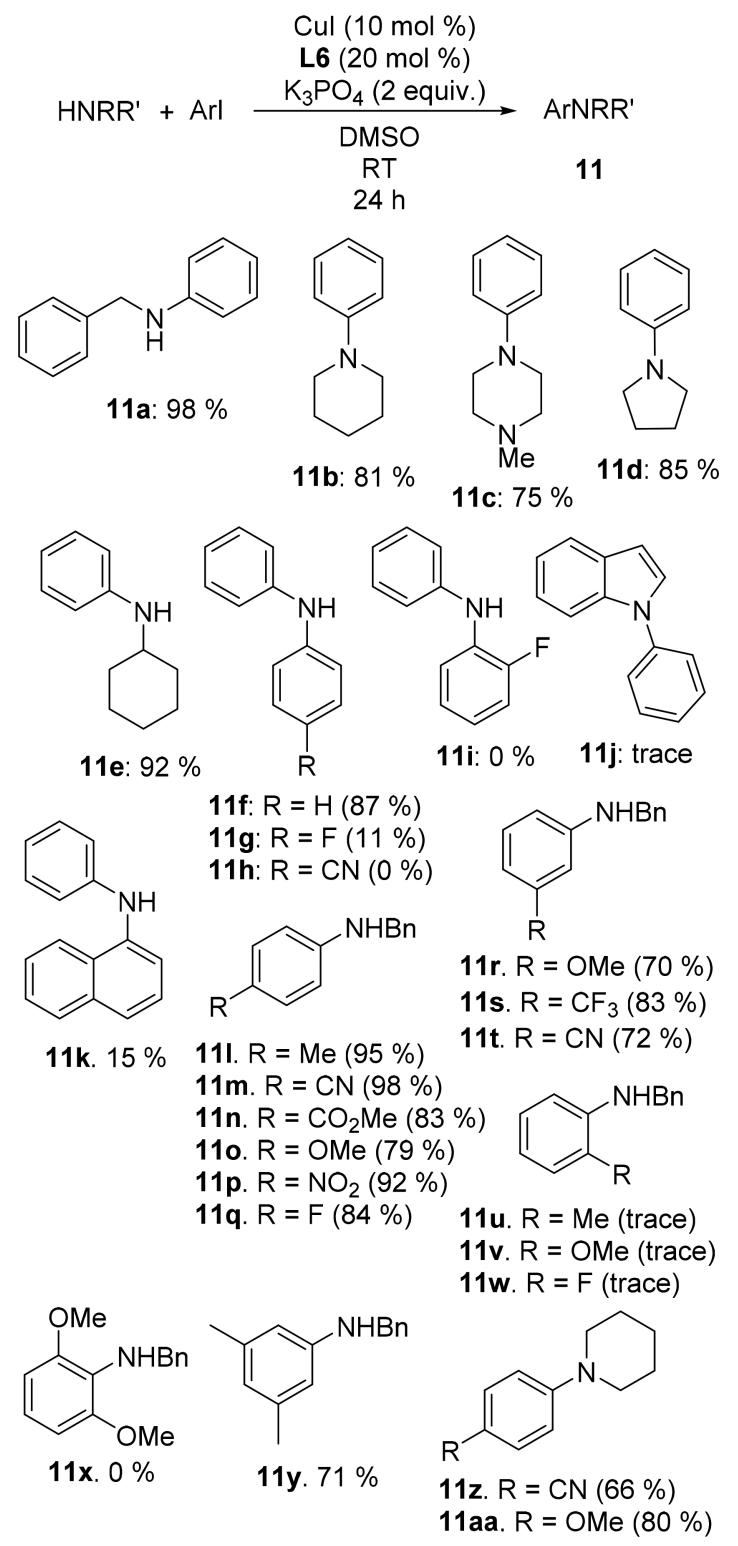

${ }^{a}$ Reaction conditions: HNRR' (1.5 mmol), ArI ( $\left.1 \mathrm{mmol}\right)$, CuI $(0.1$ $\mathrm{mmol}), \mathrm{N}$-methylglycine $(0.2 \mathrm{mmol}), \mathrm{K}_{3} \mathrm{PO}_{4}(2 \mathrm{mmol})$, DMSO (1 $\mathrm{mL})$. Isolated yields are reported.

of catalyst deactivation in particular are reported to vary considerably among the different auxiliary ligands. Together these data provide a much better insight into the relative merits of the different ligands in comparison to the final yield measurements which are usually cited.

Further kinetic, spectroscopic, and experimental studies strongly suggest that the on-cycle mechanism of $\mathrm{C}-\mathrm{N}$ bond formation (namely, coordination of the amine to the copper(I), deprotonation of the amine, oxidative addition of the aryl iodide, and finally reductive elimination) is consistent across all auxiliary ligand systems. However, additional off-cycle processes have been observed for some auxiliary ligands, most notably phenanthroline (L4), which readily forms a very stable but dormant diionic species. In addition, kinetic rate experiments reveal the ability of the auxiliary ligand, base (malonate dianion), and substrate (amine) to all act competitively as ligands for the copper center. This adds significantly to the complexity of these systems, making optimization and modeling all the more challenging.

Nevertheless, on the basis of our studies $N$-methylglycine (L6) was identified to possess the best balance of high catalytic reaction rates and low catalyst deactivation for room-temperature $\mathrm{C}-\mathrm{N}$ couplings. Hence, a new and efficient roomtemperature Ullmann amination reaction system was designed where the combination of $\mathrm{K}_{3} \mathrm{PO}_{4}$ and $\mathrm{N}$-methylglycine (L6) gave excellent reactivity with high room-temperature yields reported for a variety of amines and aryl iodides (27 examples in total). We are currently using a similar methodology to explore other copper-catalyzed aminations involving more challenging substrates with lower catalyst and auxiliary ligand loadings.

\section{ASSOCIATED CONTENT}

\section{Supporting Information}

The Supporting Information is available free of charge on the ACS Publications website at DOI: 10.1021/acscatal.7b03664.

Experimental procedures, experimental and spectroscopic data, full graphs related to the kinetic studies, and details of the kinetic modeling using COPASI (PDF)

\section{AUTHOR INFORMATION}

\section{Corresponding Author}

*R.P.D.: e-mail, r.davies@imperial.ac.uk; tel, +44 (0)207 5945754.

ORCID $\odot$

D. Christopher Braddock: 0000-0002-4161-7256

Robert P. Davies: 0000-0003-1503-0969

Notes

The authors declare no competing financial interest.

\section{ACKNOWLEDGMENTS}

This work was supported by Syngenta and the UK EPSRC (Grant EP/M507878/1).

\section{REFERENCES}

(1) Ullmann, F. Ber. Dtsch. Chem. Ges. 1903, 36, 2382-2384.

(2) Goodbrand, H. B.; Hu, N. X. J. Org. Chem. 1999, 64, 670-674.

(3) Zhang, H.; Cai, Q.; Ma, D. J. Org. Chem. 2005, 70, 5164-5173.

(4) Lin, H.; Sun, D. Org. Prep. Proced. Int. 2013, 45, 341-394.

(5) Fischer, C.; Koenig, B. Beilstein J. Org. Chem. 2011, 7, 59-74.

(6) Bedos-Belval, F.; Rouch, A.; Vanucci-Bacqué, C.; Baltas, M. MedChemComm 2012, 3, 1356-1372.

(7) Altman, R. A.; Koval, E. D.; Buchwald, S. L. J. Org. Chem. 2007, $72,6190-6199$

(8) Ma, D.; Cai, Q.; Zhang, H. Org. Lett. 2003, 5, 2453-2455.

(9) Yang, C.-T.; Fu, Y.; Huang, Y.-B.; Yi, J.; Guo, Q.-X.; Liu, L. Angew. Chem., Int. Ed. 2009, 48, 7398-7401.

(10) Ma, D.; Liu, F. Chem. Commun. 2004, 3, 1934-1935.

(11) Shafir, A.; Buchwald, S. L. J. Am. Chem. Soc. 2006, 128, 87428743.

(12) Shafir, A.; Lichtor, P. A.; Buchwald, S. L. J. Am. Chem. Soc. 2007, 129, 3490-3491.

(13) Evano, G.; Blanchard, N.; Toumi, M. Chem. Rev. 2008, 108, 3054-3131.

(14) Evano, G.; Toumi, M.; Coste, A. Chem. Commun. 2009, 41664175.

(15) Ma, D.; Xia, C.; Jiang, J.; Zhang, J.; Tang, W. J. Org. Chem. 2003, $68,442-451$.

(16) Ma, D.; Zhang, Y.; Yao, J.; Wu, S.; Tao, F. J. Am. Chem. Soc. 1998, 120, 12459-12467. 
(17) Ma, D.; Xia, C.; Jiang, J.; Zhang, J. Org. Lett. 2001, 3, 21892191.

(18) Hartwig, J. F. Acc. Chem. Res. 1998, 31, 852-860.

(19) Wolfe, J. P.; Wagaw, S.; Marcoux, J.-F.; Buchwald, S. L. Acc. Chem. Res. 1998, 31, 805-818.

(20) Hartwig, J. F. Pure Appl. Chem. 1999, 71, 1417-1423.

(21) Yang, B. H.; Buchwald, S. L. J. Organomet. Chem. 1999, 576, $125-146$.

(22) Sperotto, E.; van Klink, G. P. M.; van Koten, G.; de Vries, J. G. Dalt. Trans. 2010, 39, 10338-10351.

(23) Sambiagio, C.; Marsden, S. P.; Blacker, A. J.; McGowan, P. C. Chem. Soc. Rev. 2014, 43, 3525-3520.

(24) Monnier, F.; Taillefer, M. Angew. Chem., Int. Ed. 2008, 47, 3096-3099.

(25) Monnier, F.; Taillefer, M. Angew. Chem., Int. Ed. 2009, 48, 6954-6971.

(26) Casitas, A.; Ribas, X. Chem. Sci. 2013, 4, 2301-2318.

(27) Gurjar, K. K.; Sharma, R. K. ChemCatChem 2017, 9, 862-869.

(28) Rovira, M.; Jašíková, L.; Andris, E.; Acuña-Parés, F.; Soler, M.; Güell, I.; Wang, M.-Z.; Gómez, L.; Luis, J. M.; Roithová, J.; Ribas, X. Chem. Commun. 2017, 53, 8786-8789.

(29) Blackmond, D. G. Angew. Chem., Int. Ed. 2005, 44, 4302-4320.

(30) Blackmond, D. G. J. Am. Chem. Soc. 2015, 137, 10852-10866.

(31) Strieter, E. R.; Blackmond, D. G.; Buchwald, S. L. J. Am. Chem. Soc. 2005, 127, 4120-4121.

(32) Strieter, E. R.; Bhayana, B.; Buchwald, S. L. J. Am. Chem. Soc. 2009, 131, 78-88.

(33) Sung, S.; Sale, D.; Braddock, D. C.; Armstrong, A.; Brennan, C.; Davies, R. P. ACS Catal. 2016, 6, 3965-3974.

(34) Sherborne, G. J.; Adomeit, S.; Menzel, R.; Rabeah, J.; Brückner, A.; Fielding, M. R.; Willans, C. E.; Nguyen, B. N. Chem. Sci. 2017, 8, 7203-7210.

(35) Ruiz-Castillo, P.; Buchwald, S. L. Chem. Rev. 2016, 116, 1256412649.

(36) Zhou, W.; Fan, M.; Yin, J.; Jiang, Y.; Ma, D. J. Am. Chem. Soc. 2015, 137, 11942-11945.

(37) Fan, M.; Zhou, W.; Jiang, Y.; Ma, D. Org. Lett. 2015, 17, 59345937.

(38) Fan, M.; Zhou, W.; Jiang, Y.; Ma, D. Angew. Chem., Int. Ed. 2016, 55, 6211-6215.

(39) Xia, S.; Gan, L.; Wang, K.; Li, Z.; Ma, D. J. Am. Chem. Soc. 2016, 138, 13493-13496.

(40) Gao, J.; Bhunia, S.; Wang, K.; Gan, L.; Xia, S.; Ma, D. Org. Lett. 2017, 19, 2809-2812.

(41) Pawar, G. G.; Wu, H.; De, S.; Ma, D. Adv. Synth. Catal. 2017, 359, 1631-1636.

(42) Rovira, M.; Soler, M.; Güell, I.; Wang, M.-Z.; Gomez, L.; Ribas, X. J. Org. Chem. 2016, 81, 7315-7325.

(43) Tye, J. W.; Weng, Z.; Johns, A. M.; Incarvito, C. D.; Hartwig, J. F. J. Am. Chem. Soc. 2008, 130, 9971-9983.

(44) Tye, J. W.; Weng, Z.; Giri, R.; Hartwig, J. F. Angew. Chem., Int. Ed. 2010, 49, 2185-2189.

(45) Cai, Q.; Zhang, H.; Zou, B.; Xie, X.; Zhu, W.; He, G.; Wang, J.; Pan, X.; Chen, Y.; Yuan, Q.; Liu, F.; Lu, B.; Ma, D. Pure Appl. Chem. 2009, 81, 227-234.

(46) Kwong, F. Y.; Klapars, A.; Buchwald, S. L. Org. Lett. 2002, 4, $581-584$.

(47) Otto, N.; Opatz, T. Beilstein J. Org. Chem. 2012, 8, 1105-1111.

(48) Uma Maheswar Reddy, K.; Santosh Kumar, K.; Panasa Reddy, A. Asian J. Chem. 2014, 26, 4747-4751.

(49) Sung, S.; Braddock, D. C.; Armstrong, A.; Brennan, C.; Sale, D.; White, A. J. P.; Davies, R. P. Chem. - Eur. J. 2015, 21, 7179-7192.

(50) He, C.; Zhang, G.; Ke, J.; Zhang, H.; Miller, J. T.; Kropf, A. J.; Lei, A. J. Am. Chem. Soc. 2013, 135, 488-493.

(51) Cheng, B.; Yi, H.; He, C.; Liu, C.; Lei, A. Organometallics 2015, 34, 206-211.

(52) Experiments involving higher ligand loadings could not be carried out owing to the poor solubility of amino acids in DMSO at concentrations above $20 \mathrm{~mol} \%$ at room temperature.
(53) Rannulu, N. S.; Rodgers, M. T. J. Phys. Chem. A 2007, 111, 3465-3479.

(54) Giri, R.; Hartwig, J. F. J. Am. Chem. Soc. 2010, 132, 1586015863.

(55) Annunziata, A.; Galli, C.; Marinelli, M.; Pau, T. Eur. J. Org. Chem. 2001, 2001, 1323-1329.

(56) Deldaele, C.; Evano, G. ChemCatChem 2016, 8, 1319-1328. 